\title{
Libros de texto en el Instituto Nacional de Gestión Sanitaria (INGESA)
}

\author{
Pilar DEL CAMPO PUERTA \\ Doctora en Ciencias de la Documentación \\ campopuerta@gmail.com
}

Recibido: Febrero 2015

Aceptado: Abril 2015

\begin{abstract}
Resumen: Los libros de texto una vez realizada su función de enseñanza suelen caer en el olvido y su salvación depende, casi en exclusiva, del aprecio y consideración de sus poseedores; luego, en la mayoría de las ocasiones, esas colecciones privadas pasan por donación a bibliotecas de instituciones donde se custodian como patrimonio bibliográfico. El Instituto Nacional de Gestión Sanitaria (INGESA) es una de las Instituciones ubicadas en Madrid donde en su biblioteca se conservan libros de texto del siglo XIX. Teniendo en cuenta que en los textos escolares de ese periodo se hicieron muchas y sucesivas ediciones con cambios en el título, extensión, etc., lo que añade un problema a la catalogación, los títulos que se van a analizar pertenecen a la lista de libros autorizados, por diversos Reales Decretos, para los alumnos que cursaban estudios de educación primaria y educación secundaria en colegios e institutos.
\end{abstract}

Palabras clave: Aritmética; Bibliotecas madrileñas; Educación primaria; Educación secundaria; Estudios; Ética; Geografía; Historia natural; Instituto de Gestión Sanitaria; Libros de texto; Matemáticas; siglo XIX.

\section{Textbook in Instituto Nacional de Gestión Sanitaria (INGESA)}

\begin{abstract}
Textbooks, once they have fulfilled their function of teaching, are forgotten and their salvation depends, almost exclusively, on the appreciation and consideration of their holders; then, in most cases, some private it is collections are donated to institutions and public libraries which are kept bibliographic heritage. Instituto Nacional de Gestión Sanitaria (INGESA) is the Health Management Institute, one institution located in Madrid where textbooks of the nineteenth Century are preserved in their library. Given that many successive editions were made with changes in title, length, etc. in textbooks of that period, which adds a problem to cataloging the titles that will be analyzed belong to the list of books authorized by various Real Decrees, for students who were studying in primary and secondary education in schools and colleges.
\end{abstract}

Keywords: Arithmetic; Madrid libraries; Primary education; Secondary education; Studies; Ethics; Geography; Natural history; Health Management Institute; Textbooks; Mathematics; Nineteenth century. 


\section{INTRODUCCIÓN}

La importancia de los fondos bibliográficos depende no sólo de su valor intrínseco, sino también de la utilidad y disfrute social que puedan proporcionar; por ello, "Todos los poseedores de bienes del Patrimonio Documental y Bibliográfico están obligados a conservarlos, protegerlos, destinarlos a un uso que no impida su conservación y mantenerlos en lugares adecuados” (Art. 52, Ley 16/1985, de 25 de junio de Patrimonio Histórico Español) ${ }^{1}$

Los libros de texto antiguos, que forman parte del patrimonio documental y bibliográfico, una vez realizada su función de enseñanza suelen caer en el olvido y su salvación depende, casi en exclusiva, del aprecio y consideración de sus poseedores siendo las colecciones privadas quienes recogen esa nostalgia y evocación al pasado escolar; no así ha ocurrido en las bibliotecas más generales de titularidad pública, pues como Alejandro Tiana Ferrer dice, "apenas se han producido esfuerzos sistemáticos tendentes a su conservación" (Tiana Ferrer, 1999: 101-119)²; aunque también es cierto que estos libros son cada vez más atractivos para los investigadores, lo que aviva el interés de conservación. No obstante, hay que tener en cuenta que en los textos escolares del siglo XIX se hicieron muchas y sucesivas ediciones con cambios en el título, extensión, etc., lo que añade un problema a la catalogación.

Como pone de manifiesto Alain Choppin, "los manuales escolares pueden ser estudiados desde distintos puntos de vista, ya que son a un tiempo productos de consumo, soporte de conocimientos escolares, vectores ideológicos y culturales e instrumentos pedagógicos"1 (Choppin, 1992: 102), por ello, se inicia una serie de artículos para estudiar este patrimonio documental teniendo como base la tesis Materiales didácticos de los siglos XVIII y XIX en bibliotecas madrileñas ${ }^{3}$ y siendo la Biblioteca del Instituto de Gestión Sanitaria (INGESA) ${ }^{4}$ la primera a analizar para conocer más a fondo los libros de texto que se conservan, analizando cada uno de ellos conforme a la metodología establecida de búsqueda de la

${ }^{1}$ ESPAÑA. Ley 16/1985, de 25 de junio de Patrimonio Histórico Español. Boletín Oficial del Estado, de 29 de junio de 1985, nº 155, pp. 20342-20352,

$<$ https://www.boe.es/diario_boe/txt.php?id=BOE-A-1985-12534> [Consulta: 30/10/2014].

2 TIANA FERRER, Alejandro (1999) "La investigación histórica sobre los manuales escolares en España: el proyecto MANES” en Clio \& Asociados. La historia enseñada, $\mathrm{n}^{\circ}$ 4, pp. 101-119, $<$ http://sedici.unlp.edu.ar/bitstream/handle/10915/32114/Documento_completo.pdf?sequence=1> [Consulta: 30/01/2012].

${ }^{3}$ CAMPO PUERTA, M ${ }^{\text {a }}$ Pilar del. "Materiales didácticos de los siglos XVIII y XIX en bibliotecas madrileñas” Director: José Luis Gonzalo Sánchez-Molero, Universidad Complutense de Madrid, Departamento de Filología, 2014.

${ }^{4}$ En el año 2002, finalizadas las transferencias sanitarias a las Comunidades Autónomas, el INSALUD se convierte en una nueva entidad que pasa a denominarse Instituto Nacional de Gestión Sanitaria (INGESA). 
información en tres procesos diferentes: 1) considerando las listas de libros aprobados y censurados para el estudio según la legislación vigente del momento, reunida de manera exacta y minuciosa por Villalaín Benito (Villalaín Benito, 1999) $)^{5}$ en su obra Manuales escolares en España (Tomos I, II y III), 2) búsquedas de datos en el Catálogo Colectivo del Patrimonio Bibliográfico Nacional (CCPB) y 3) analizando el propio documento, como se verá más adelante.

\section{LA BIBLIOTECA DEL INSTITUTO NACIONAL DE GESTIÓN SANITARIA (INGESA)}

La Biblioteca, que tiene su origen en la Biblioteca del antiguo Instituto Nacional de Previsión (INP), fue fundada en 1908 para cubrir las necesidades culturales, científicas y de difusión del propio INP. Su fondo bibliográfico data de los siglos XVIII y XIX y es procedente del Palacio de Portugalete, edificio emblemático en Madrid que fue demolido por las continuas remodelaciones urbanísticas.

El actual palacete se construyó en la segunda mitad del siglo XIX, pero en la década de los años 40 del siglo XX el Palacio fue vendido al Estado para ubicar en él al Instituto Nacional de Previsión (INP) ${ }^{6}$; la venta incluía mobiliario, enseres, maderas nobles, esculturas, accesorios y libros. Es así como la biblioteca del Instituto se hace con un destacado fondo bibliográfico de obras sobre Historia, Literatura y Filosofía.

Este palacio fue residencia del tercer Duque de Bailén, Eduardo Corondelet y Donado (1820-1882), que también ostentaba los títulos de Barón de Corondelet y Marqués de Portugalete ${ }^{7}$, gran mecenas de los artistas de su tiempo, que reunió una notable colección de cuadros, lámparas, estatuas y libros.

Residente también del Palacio de Portugalete fue Camilo Hurtado de Amézaga y Balmaseda, Marqués de Riscal (1827-1888), por lo que ambas colecciones se mezclan, aunque son fácilmente identificables ya que las del Marqués de Riscal llevan un Ex libris que las identifica con las siguientes anotaciones de nombre (C. $\mathrm{H}$. de Amézaga), armario, estante, fila $\mathrm{y}^{\mathrm{o}}$ de registro, lo que muestra la escrupulosidad con la que dicha colección estaba ordenada.

Respecto al Fondo Antiguo, entre 1700 y 1900, son cuatro los fondos que se diferencian: Biblioteca Camilo Hurtado de Ámezaga, el más amplio con 617 títulos (aunque el fondo está compuesto por 900 títulos en total); Biblioteca del Duque de Bailén, para las mismas fechas, con 206 títulos (aunque sean 241en

${ }^{5}$ VILLALAÍN BENITO, José Luis, (1999) Manuales escolares en España. Tomo I. Legislación (1812-1939); Tomo II : Libros de textos autorizados y censurados (1833-1874); Tomo III : Libros de textos autorizados y censurados (1874-1939). Madrid, Universidad Nacional de Educación a Distancia.

${ }^{6}$ Actual sede del INGESA, que conserva todo lo heredado.

${ }^{7}$ De aquí el nombre que recibe el palacio, Palacio de Portugalete. 
total); Biblioteca Legado Sancho, para las mismas fechas, con 120 títulos (siendo 206 en total). Y cuarto fondo infantil, aunque dentro del Fondo Amézaga, con 59 libros de literatura infantil en inglés y francés pertenecientes a María Milagro Hurtado de Amézaga, nieta del Marqués de Riscal.

Respecto a la visibilidad de la biblioteca dentro de la página web del Instituto Nacional de Gestión Sanitaria, hay que decir que no es directa, pues para acceder a ella debe hacerse por el apartado Estadísticas y Estudios.

\section{LIBROS APROBADOS Y CENSURADOS PARA EL ESTUDIO}

En 1825, dentro de los llamados Planes de Calomarde ${ }^{8}$ aparece la recomendación de que se impartan en todas las escuelas del reino, sin distinción de clase o categoría, las mismas materias y se utilicen iguales textos. Con posterioridad y siguiendo una breve cronología explicativa, hasta llegar a un control oficial de libros para el estudio, se debe señalar que a partir de 1833 empiezan a regularse las obras recomendadas y de utilidad pública para la enseñanza; en 1836, el Plan General de Instrucción Pública ${ }^{9}$, conocido como Plan Rivas, recoge en su Art. 84 que la lengua nacional sería la única que se usara en las explicaciones y libros de texto (también en su Art. 85 referido a los Institutos superiores, menciona que hay libertad para elegir los libros de estudios, pues los profesores no estaban sujetos a seguir normas en sus explicaciones ni podían imponerla a los alumnos); en 1838 y según la ley de Instrucción primaria ${ }^{10}$, los libros de textos quedaron sometidos a la aprobación de las respectivas comisiones provinciales; en 1840 y conforme al Reglamento de Montesino" ${ }^{11}$ "No se designarán en lo sucesivo libros determinados, sino que serán elegidos por el maestro, de acuerdo con la Comisión local, las mejores obras a medida que vayan publicándose. Deberán, sin embargo, las Comisiones locales dar conocimiento a las de provincia, sin cuya aprobación no continuará el uso de libro alguno". También, en 1845 y según el Plan Pidal ${ }^{12}$ : “[...] [los] libros que deben servir de texto. Desde el arreglo provisional de 1836 prevaleció el sistema de dejar al profesor entera libertad para elegirlos. Sin examinar ahora la bondad absoluta de este sistema, lo cierto es que su adopción ha sido prematura en España, y sus

\footnotetext{
${ }^{8}$ LÓPEZ DEL CASTILLO, M ${ }^{a}$ Teresa, (1982) “Planes y programas escolares en la legislación española”. BORDÓN no 242-243. Madrid, Sociedad Española de Pedagogía.

9 Plan General de Instrucción Pública <http://www.filosofia.org/mfa/fae836a.htm\#00> [Consulta: 30/10/2014].

${ }^{10}$ Ministerio de Educación (1979) "Colección Legislativa de Instrucción Primaria. Madrid, 1856” en Ministerio de Educación y Ciencia (1979) Historia de la Educación en España. Textos y Documentos. Vol II: De las Cortés de Cádiz a la revolución de 1868. Madrid, Ministerio de Educación.

${ }^{11}$ Ibídem.

12 Plan General de Instrucción Pública. Real Decreto de 17 de septiembre de 1845. [en línea]. Disponible en http://www.filosofia.org/mfa/fae845a.htm [Consulta: 30/10/2014].
} 
resultados, nada favorables. Ejemplos se han visto verdaderamente escandalosos de catedráticos que, abusando de esta libertad, han señalado textos que por su antigüedad, su descrédito o su ninguna conexión con el objeto de la asignatura, más bien que de enseñanza servían a los jóvenes de errada y funesta guía. Verdad es que cuando el Gobierno prescribe los libros de enseñanza, entra el recelo de que tienda a comprimir las ideas o establecer un monopolio exclusivo en favor de autores determinados. El proyecto, huyendo de todos estos extremos, establece que el Consejo de Instrucción Pública forme para cada asignatura una lista corta de obras selectas, entre las cuales pueda elegir el catedrático la que mejor le parezca, y que esta lista sea revisada por la misma corporación cada tres años [...]”.

Pero es por la Real Orden de 22 de agosto de 1846 cuando se aprueban las primeras listas de obras de texto presentadas por el Consejo de Instrucción pública, siendo en total 47 listas entre obras aprobadas y jusrispreciadas y las obras no aprobadas o censuradas para la instrucción primaria ${ }^{13}$, desde 1847 a 1859, teniendo en cuenta, además, que según R. O. 21 de octubre de 1856: "Los maestros que adopten libros no aprobados, y los inspectores que lo consientan, incurrirán en la responsabilidad que haya lugar, ${ }^{\text {, }}$

Ese mismo sistema de aprobación y censura se llevó a la segunda enseñanza y a las Facultades como muestra la Ley de Instrucción Pública de 9 de septiembre de 1857, también conocida como del Plan Moyano, ${ }^{15}$ que en su Título V sobre los libros de texto, dice: "Todas las asignaturas de la primera y segunda enseñanza, las de las carreras profesionales y superiores y las de las facultades hasta el grado de Licenciado, se estudiarán por libros de texto: estos libros serán señalados en listas que el Gobierno publicará cada tres años” (Art. 86).

De las citadas listas, según la tesis mencionada de Materiales didácticos de los siglos XVIII y XIX en bibliotecas madrileñas se han localizado muchos libros de textos; en este caso, varios en la biblioteca del Instituto de Gestión Sanitaria (INGESA), utilizando el Catálogo Colectivo del Patrimonio Bibliográfico (CCPB) como instrumento principal de búsqueda. Y como se verá en el siguiente apartado, en cada una de las obras que se analizan se incorpora la información legal de aprobación de las mismas por Real Orden.

${ }^{13}$ VILLALAÍN BENITO, José Luis (1999) Manuales escolares en España : Tomo II : Libros de textos autorizados y censurados (1833-1874). Madrid, Universidad Nacional de Educación a Distancia. Ob. Cit.

${ }^{14}$ Boletín oficial del Ministerio de Fomento (1856). <https://books.google.es/books?id=EX5lclWP-EC\&printsec=frontcover\&hl=es\&source=gbs_ge_summary_r\&cad=0\#v=onepage \&q\&f=false $>$

[Consulta: 30/10//2014].

15 ESPAÑA. Ley de Instrucción Pública, de 9 de septiembre de 1857. <http://personal.us.es/alporu/historia/ley_moyano_texto.htm> [Consulta: 30/10/2014]. 


\section{LIBROS DE TEXTO LOCALIZADOS. ANÁLISIS PARTICULAR DE CADA UNO}

Conforme a la metodología y búsqueda de información por los procesos citados, los libros a analizar son: Curso de Matemáticas de José María de Odriozola, Elementos de Ética de José María Rey y Heredia, Elementos de Geografía astronómica y física de Antonio Montenegro, Fábulas ascéticas de Cayetano Fernandez, Manual de Historia natural de Manuel María José de Galdo y Tratado de Aritmética de Mr. Lacroix, traducido por Rebollo.

4.1 Curso de Matemáticas, de José María de Odriozola.

También queda citado como Curso completo de Matemáticas, Curso completo de Matemáticas puras y Curso elemental de Matemáticas; para la Secundaria y aprobado por R.O. 22 agosto 1846, 8 septiembre 1847, 14 septiembre 1848, 22 septiembre 1849, 26 septiembre 1850, 5 septiembre 1851, 18 septiembre 1853, 15 septiembre 1852, 13 octubre 1854 y 1 octubre 1855.

Los tres ejemplares que siguen forman parte de una colección de cuatro tomos, que si bien se localizan en el CCPB, 158520-7, con una signatura anterior o antigua, en el INGESA figuran con otra más actualizada desde que se llevó a cabo la reorganización del espacio. Dichas signaturas del Fondo Antiguo ahora son para el Tomo II, sig. 000568 (antigua 021685); Tomo III, sig. 000569 (antigua 021686) y Tomo IV, sig. 000569 (antigua 021687). Encuadernación en pasta y bien conservados. Proceden de la Biblioteca del Duque de Bailén.

Figura 1. Portada de Geometría Elemental

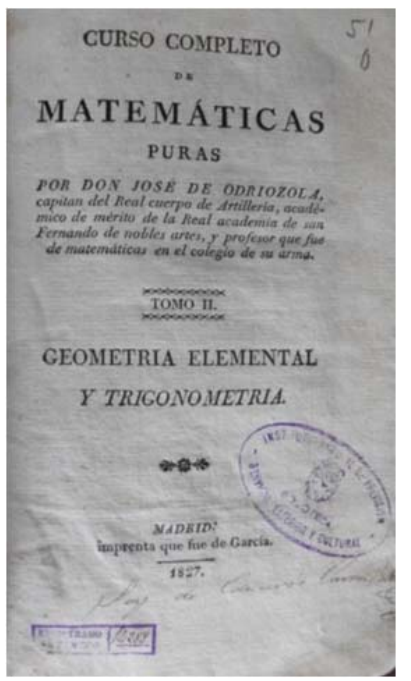

Figura 2. Portada de Algebra Sublime

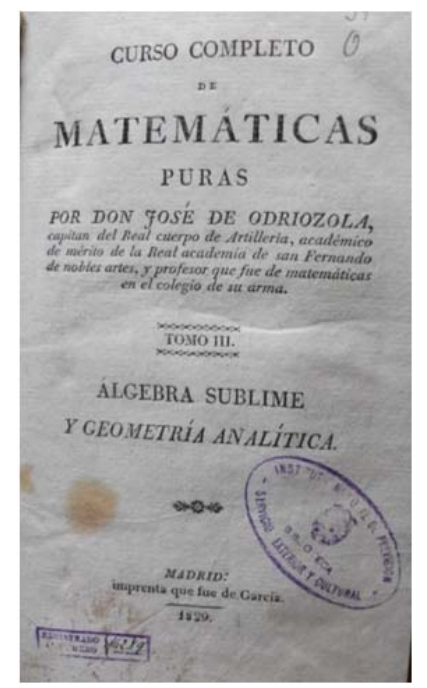


Figura 3. Portada de Cálculo diferencial

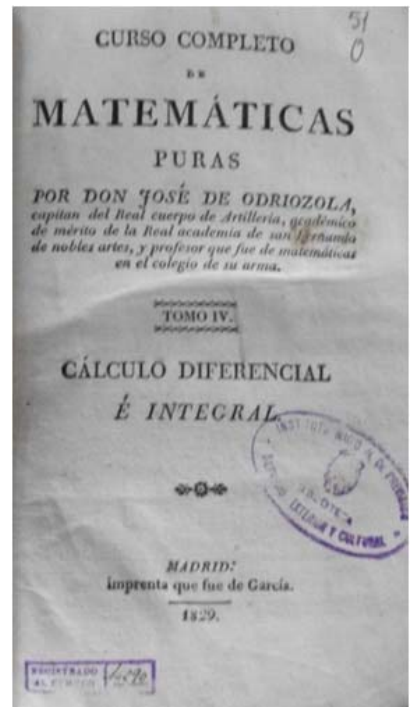

Figura 4. Portada de Geometría Elemental

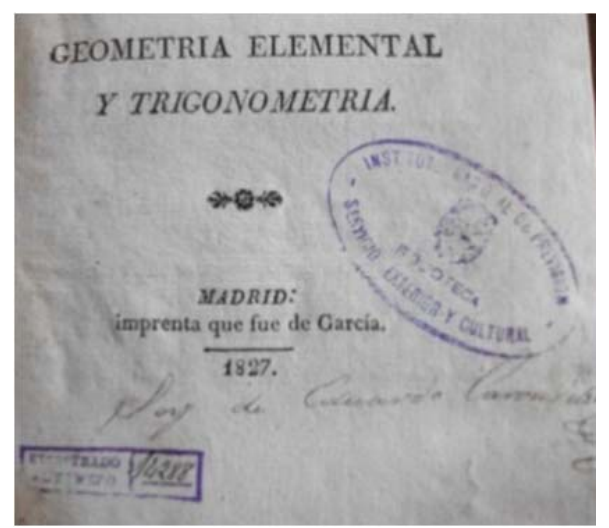

Odriozola, José de (1785-1864)

Curso completo de matemáticas puras / por el Teniente Coronel D. José de Odriozola.- Madrid : Imprenta que fue de García, 1827-1833

3 v. (385, [1] p., 18 h de lám. pleg. ; 344 p., 6 h. de lám. pleg. ; 348, [4] p., 3 h de lám. pleg.) ; $20 \mathrm{~cm}$.

En tomo II y III anotaciones manuscritas: "Soy de Eduardo Carondelet" ${ }^{\text {, }}$, y en Tomo III, p. 342 “Alcalá de Henares”.

Contiene: Tomo II: Geometría elemental y trigonometría - Tomo III: Algebra sublime y geometría - Tomo IV: Cálculo diferencial e integral.

Las h. de grab. pleg. son grabs. calcográficos representando figuras matemáticas.

Localizaciones según CCPB: Almadén (Ciudad Real). Universitaria. Escuela de Ingeniería Minera e Industrial. FA-394 [Sólo t. III].- Astorga (León). Convento Padres Redentoristas. 12/C 109 [Sello de los Padres Redentoristas.- Biblioteca. Astorga.- Sello de Congregatio Sanctissimi Redemptoris. Domus Asturicensis.Sólo tomo II]; Seminario. FA.9641-9643 [Contiene únicamente los tomos II, III y IV].- Azpeitia (Guipúzcoa). Santuario de Loyola. 3071,4-28(1/4) v.2 [Ex-libris de "Biblioteca de D. Luis Zurbano".- Sólo tomo II].- Badajoz. Real Sociedad

${ }^{16}$ La información de la anotación manuscrita que se recoge en CСPB es: "Soy de Eduardo Carrondelet y Donado”; es decir, hay un error en el apellido de Eduardo Carondelet y Donado III duque de Bailén, (1820-1882). 
Económica Extremeña de Amigos del País. 53-77, 53-78, 53-79 [Falto de tomo I].Barcelona. Pública Episcopal (Seminario conciliar). 94419 [Ex-l. de Juan Lopez].- Cádiz. Biblioteca Pública. XIX-2408 [Contiene Tomo IV: Calculo Diferencial é Integral: 348 p. [3] h. de grab.- Olim: 29-644].- Granada. Instituto de Bachillerato Padre Suárez. DM-70(IV) [Sólo tomo IV]; Universidad. BHR/B026-138, BHR/B-026-139, BHR/B-026-140 [Sólo t. II, III, IV].- Javalí Viejo (Murcia). Fábrica de Pólvora (Empresa Nacional Santa Bárbara). 9-1-13 [Contiene únicamente el t. IV].- Las Palmas de Gran Canaria. Biblioteca Insular. FG-76-1-16 [Sólo Tomo II.- Ejemplar perteneciente al Fondo Particular de Fernando González].- Madrid. Central Militar (Instituto de Historia y Cultura Militar). VII-4833 [Sólo tomo IV]; VII-4833(bis) [Sólo tomo IV]; SM-18278/SM-1827-10, SM-1829-8 [Ex-libris del Museo de Literatura Militar.- Ex-libris y sello del Archivo Facultativo de Artillería.- Sellos de ¿Pirotecnia Militar de Sevilla. Artillería? y ¿Biblioteca de Tercera Clase. Pirotecnia Militar? en t. IV Olim: ML-R-117-A, ML-R-118-A]; VII-4437 [Sólo t. III]; III-27-9-7 [Ex-libris de la Biblioteca de la Academia de Ingenieros.- Sólo t. II]; III-43-10-24(bis) [Sólo tomo IV]; Biblioteca Central de la Marina (Cuartel General de la Armada) . P.460243 [Sólo t. II]; P.46-1045 [Sólo t. III]; Escuela de Guerra. 22C-5-14032(4) [Superlibris del Depósito de la Guerra.- Sólo tomo IV]; Instituto Nacional de Gestión Sanitaria. 021685-021687 [An. ms."Soy de Eduardo Carrondelet".- Falto de vol. I]; Real Academia de Bellas Artes de San Fernando. B-1872 [Sólo v. 4]; Real Academia de Ciencias Exactas, Físicas y Naturales. XIX-4544 [Sello de la Biblioteca de la Real Academia de Ciencias Exactas.- Solo vol. IV]; Real Gran Peña. FD 14-2-17(II/IV) [Legado Fernández Durán.- Falto del t. I]; Senado. 23825 [Contiene sólo el vol. 4]; Universidad Complutense, Biblioteca Histórica Marqués de Valdecilla. BH MED 14923 [Tomo 1.- Sello del Colegio de San Carlos]; BH MED 14924 [Tomo 2.- Sello del Colegio de San Carlos]; BH MED 14925 [Tomo 3.- Sello del Colegio de San Carlos]; BH MED 14926 [Tomo 4.Sello del Colegio de San Carlos].- Málaga. Biblioteca Pública. 19096 [Sólo t. III].- Oñate (Guipúzcoa). Santuario de Arantzazu. FF 1-6-1(II, III, IV) [Falto del tomo I].- Orense. Seminario Mayor. Sin sign [Solo es t. II y IV.- Donación de Jaime Rionegro].- San Fernando (Cádiz). Real Instituto y Observatorio de la Armada. 00519/00522.- Segovia. Academia de Artillería. 56-9-25050/25053 [Sello de la Biblioteca del Colegio de Artillería.- Escuela de Aplicación de Artillería.- Ex-libris ms.: "A la Biblioteca de la Academia de Artillería. 1862. El cap. Profesor Adolfo Carrasco".- Ex-libris ms.: "Eusebio Cordero"]; 56-9-25056 [Ex-libris ms.: "Regalado por la familia de D. Mariano Gil".- Ex-libris ms.: "Nicolas Jorge de Arespacochaga ..." .- Contiene T. III y IV.- Enc. 2 t. en un vol.]; 7-17-35 [Contiene únicamente T. IV]; 7-17-36 [Contiene únicamente T. IV]; 7-1735bis [Contiene únicamente T. II (385, 11 h. de lám. pleg., 7 h. de lám. pleg.)]; Biblioteca Pública. 80455 [Contiene únicamente t. IV.- Olim: 529]; 82017 [Contiene únicamente t. IV.- Olim: 2/2885]; Seminario Conciliar.11012.- Sevilla. 
Biblioteca Histórica Militar. B/5/I-3-14452 [Ex-libris de la Maestranza de Artillería.- Ex-libris de la Biblioteca Regional CGRM del sur.- Sello de la Biblioteca de Artillería de Sevilla.- Sello de la Maestranza del $3^{\circ}$ Departamento de Artillería.- Sello de la Maestranza de Artillería de Sevilla.- Sólo T. IV].- Toledo. Academia de Infantería. 51.0050-50.0051 [Sólo t. II (385 p.), t. IV (348p.)]; Biblioteca Pública. 4-16355 [Sello del Instituto de Segunda Enseñanza de la Prov. de Toledo.-Contiene únicamente t. IV].- Valencia. Provincial de las Escuelas Pías. XIX/462-465.- Zamora. Pública. ZA/8834 [Sello de la Biblioteca del Instituto de $2^{\text {a }}$ Enseñanza de Zamora.- Falto de t. II].

Separado de este grupo el Tomo I se localiza en CCPB, 236957-5, con una signatura antigua 021684, figurando en el INGESA con la signatura 000568 actual. Encuadernado en pasta presenta deterioro. También es procedente de la Biblioteca del Duque de Bailén y lleva anotaciones manuscritas.

Figura 5. Portada de Aritmética

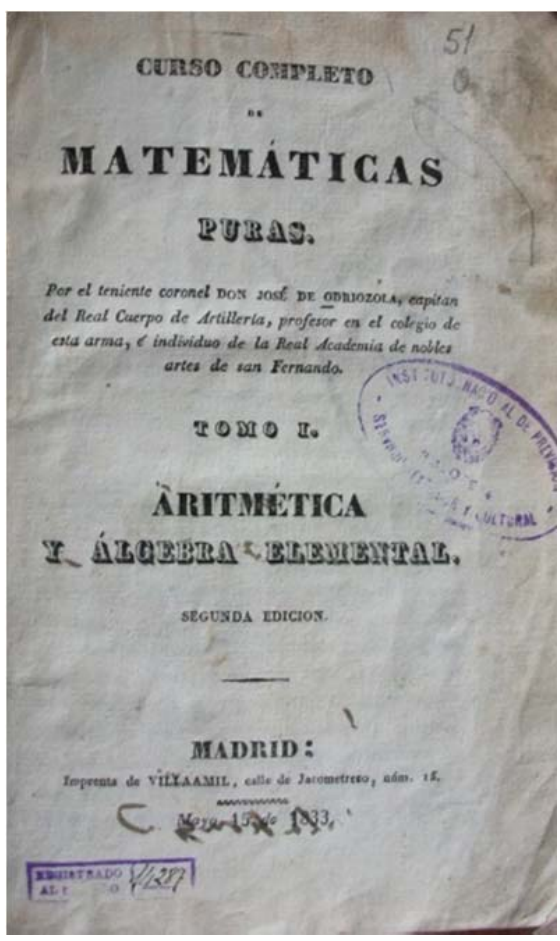

Figura 6-7. Interiores de Aritmética
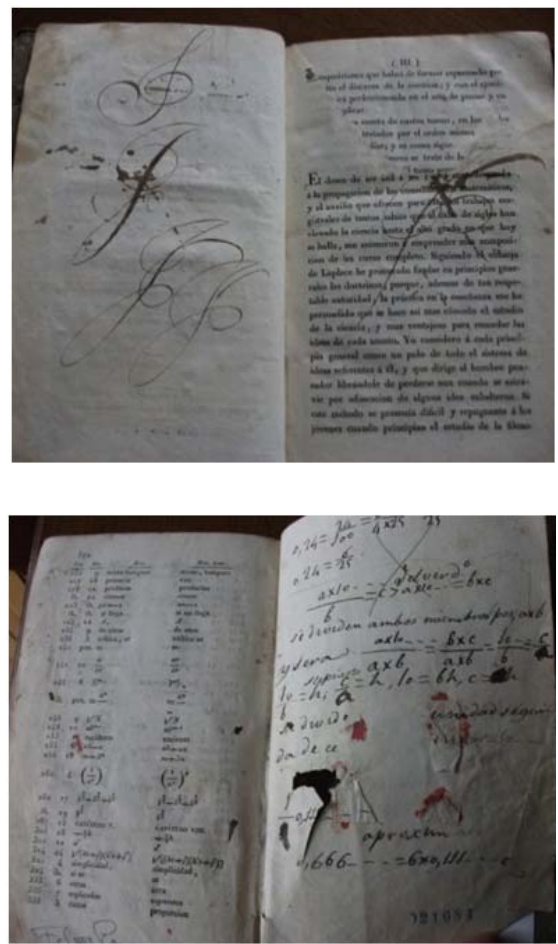

Localizaciones según CCPB: Almadén (Ciudad Real). Universidad de CastillaLa Mancha, Escuela de Ingeniería Minera e Industrial de Almadén. FA-393.Badajoz. Real Sociedad Económica Extremeña de Amigos del País. 53-76 [Sólo tomo I].- Barcelona. Biblioteca Pública Episcopal del Seminario. 94328 [Inc. vol. 
1, Sellos: "Bibliotheca Episcopalis Seminarii Barcinonensis" y "La poesia. Libreria de J. Merino ... compran, alquilan y venden libros baratos. Obispo 135. Habana" - Sólo tomo I].- Granada. Universidad. BHR/B-026-137.- Madrid. Instituto Nacional de Gestión Sanitaria. 021684; Real Gran Peña. FD 14-2-17(I) [Legado Fernández Durán.- Contiene únicamente t. I].- Málaga. Biblioteca Pública. 19095 [Sólo tomo I].- Oñate (Guipúzcoa). Santuario de Arantzazu. FF 16-1(I) [Sólo tomo I. Falto de la última p.- Este ejemplar tiene [2] pp. del índice entre la p. VI y la p. 373]; FF 1-6-2(I) [Sólo tomo I].- Oviedo. Universidad. PE50014 I.- Segovia. Academia de Artillería. 7-17-37/38.

4.2 Elementos de Ética de José María Rey y Heredia; para Secundaria y aprobado por R.O. 18 septiembre 1853, 13 octubre 1854, 1 octubre 1855, 13 septiembre 1858, 26 septiembre 1861, 31 agosto 1864, 14 septiembre 1867 y 1 agosto 1868 .

Figura 8. Portada de Tratado de Filosofía Moral

Figura 9-10. Interior y detalle
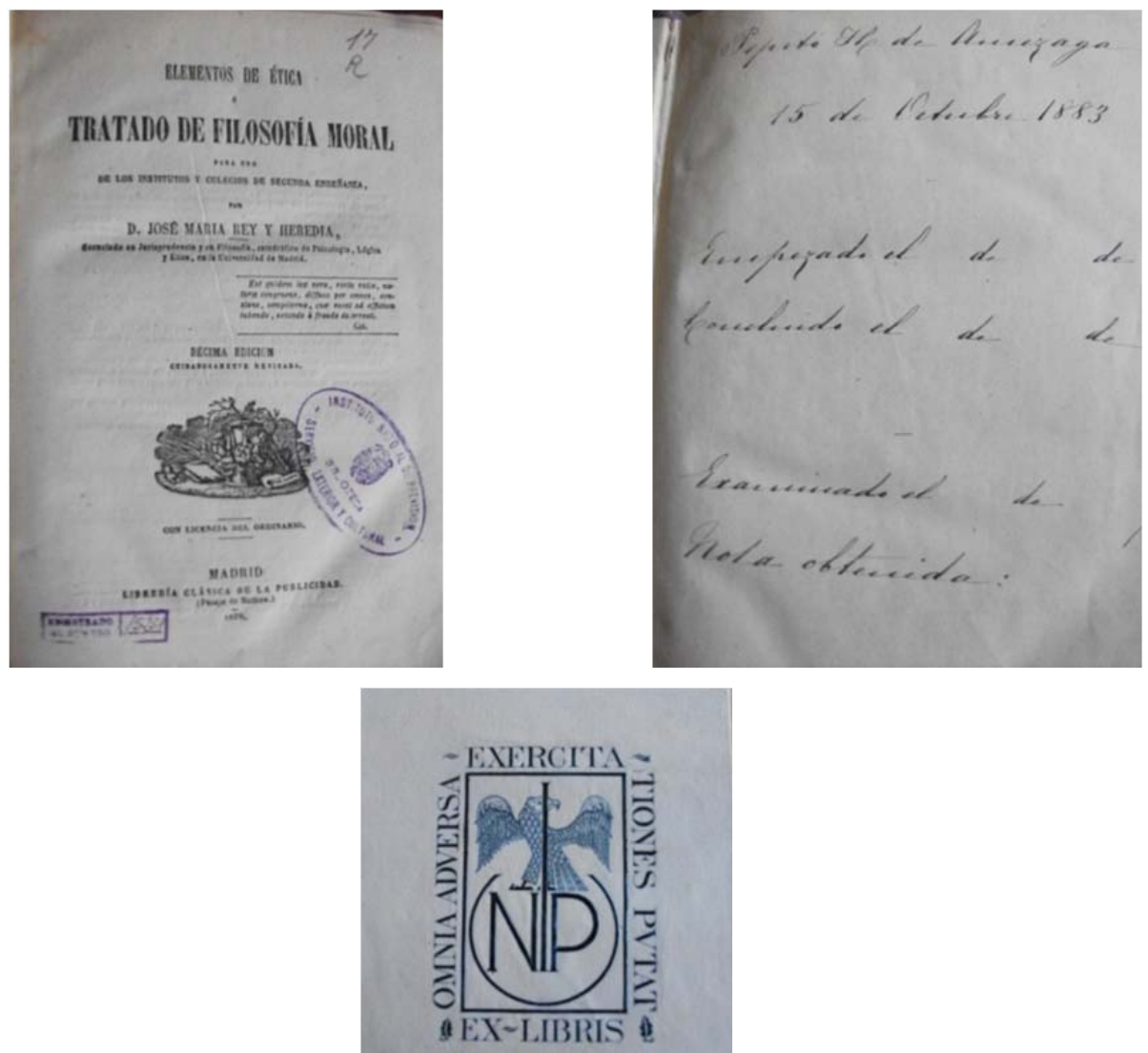
4.3 Elementos de Geografía astronómica y física, de D. Antonio Montenegro; para Secundaria y aprobado por R.O. 22 agosto 1846, 8 septiembre 1847, 14 septiembre 1848, 22 septiembre 1849 y 5 septiembre 1851.

Figura 11. Portada de Geografía

Figura 12. Interiores
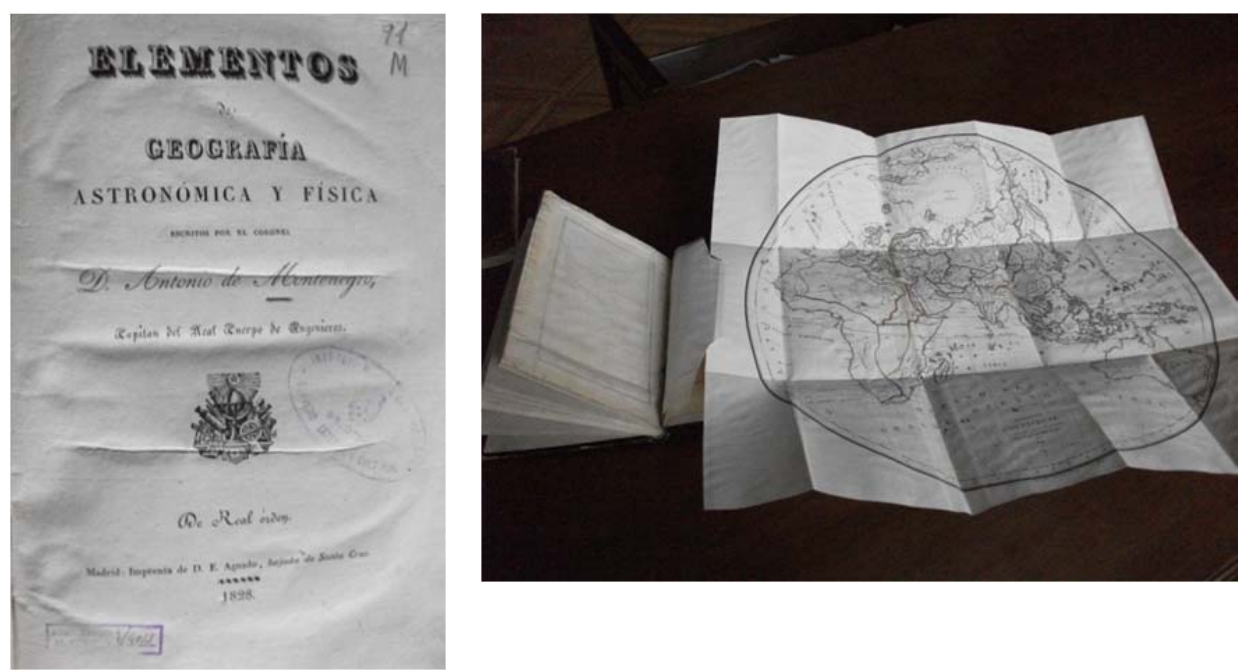

Montenegro, Antonio de.

Elementos de Geografía astronómica y física / escritos por Antonio de Montenegro.- Madrid : [s.n.], 1828 (Imprenta de D. E. Aguado). XVI, 125 p. : lám. ; 22 cm.

Las hojas de láminas calcográficas son plegadas y representan mapas y dibujos astronómicos grabados por J. B. Tardieu.

En el CCPB, 258182-5, figura con la signatura anterior o antigua, siendo la signatura actualizada del Fondo Antiguo 0001527. Procede del fondo del Marqués de Riscal, biblioteca Camilo Hurtado de Amézaga (1827-1888).

Localizaciones según CCPB: Burgo de Osma (Soria). Seminario Diocesano o Conciliar Santo Domingo de Guzmán. C-762.-Cáceres. Biblioteca Pública del Estado “A. Rodríguez Moñino y María Brey. 2/8490 [Sello de la Biblioteca Provincial de Cáceres.- Sello de Vicente Paredes y Guillén, Arquitecto de Plasencia].- Córdoba. Instituto de Educación Secundaria Séneca. 4-E-7.- Madrid. Biblioteca Central Militar (Instituto de Historia y Cultura Militar. SM-1828-5 [Ex-libris del Museo de Literatura Militar.- Sello del Archivo Facultativo de Artillería.- An. ms. "J.S.J.".- Olim: ML-R-117-A]; V-71-9-14 [Ex-libris y sello de la Biblioteca de Ingenieros]; Instituto de Gestión Sanitaria. 030757; Museo Nacional del Romanticismo. N-III-16; Real Academia de la Historia. 13/3635 
[Olim: 10-7-11; 8²-18-3/3155].- Málaga. Biblioteca Pública. 13566.- Roquetas (Tarragona). Observatorio del Ebro. H523 Mon9.- San Fernando (Cádiz). Real Instituto y Observatorio de la Armada. 04635.

4.4 Fábulas ascéticas, de Cayetano Fernandez; para Primaria y aprobado por R.O. 10 marzo 1865.

Figura 13-15. Cubierta, portada y dedicatoria de Fábulas Ascéticas
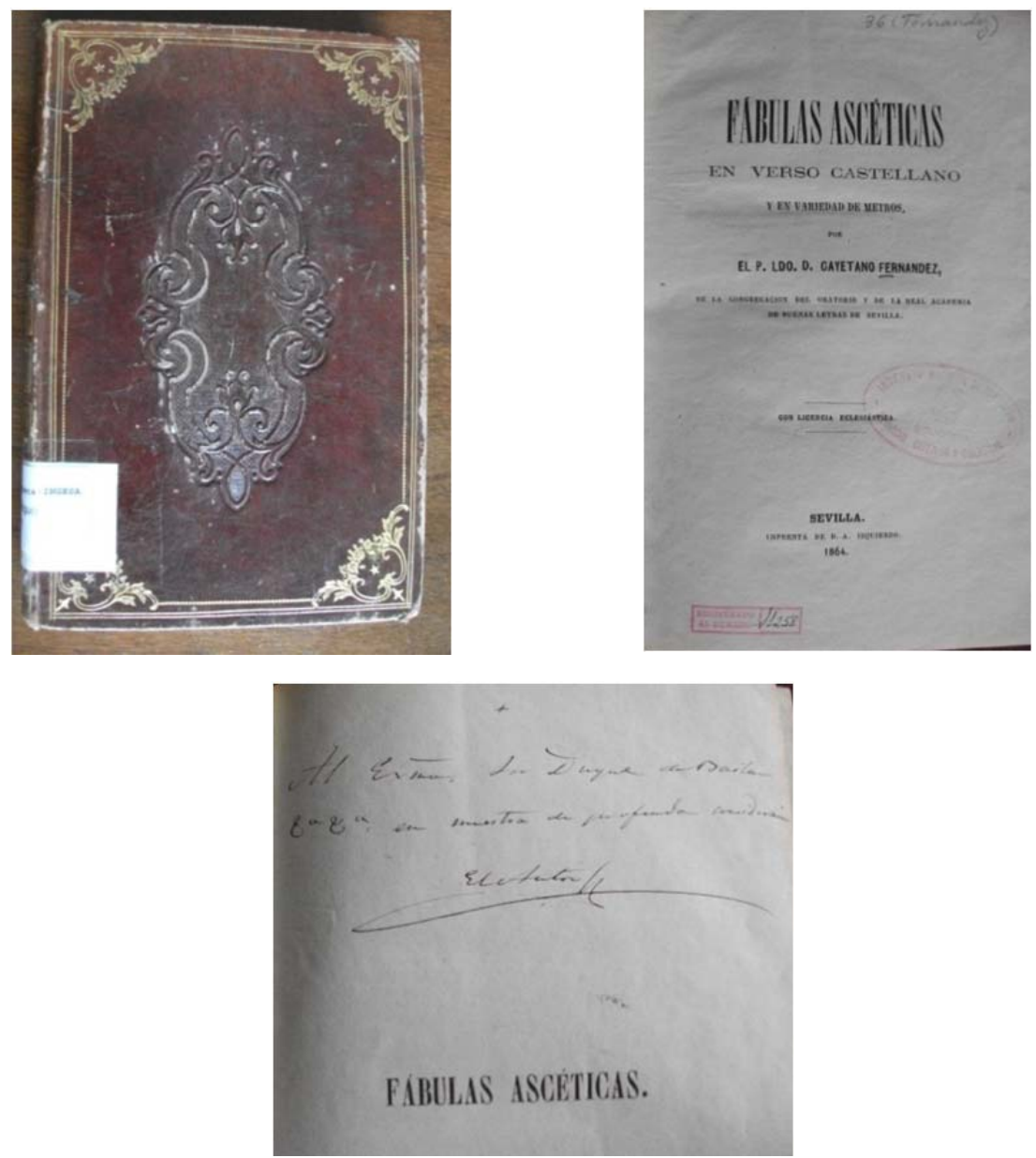

Fernández, Cayetano.

Fábulas ascéticas en verso castellano y en variedad de metros / por Cayetano Fernández.- Sevilla : [s.n.]. 1864. (Imprenta de D. A. Izquierdo). IX, 374 p. ; $21 \mathrm{~cm}$. 
Procede de la biblioteca del Duque de Bailén y en la anteportada puede leerse a dedicatoria del autor al Duque de Bailén. La signatura que puede encontrarse en CCPB, 310314-5, es la antigua 028813, siendo la signatura actual 000411. Resalta su encuadernación en piel con hierros en seco y dorado.

Las localizaciones según CCPB son: Ávila. Biblioteca Pública. Dep. 4731. [Ex-libris del Cuerpo Facultativo de Archiveros, Bibliotecarios y Arqueólogos. Biblioteca Provincial de Ávila.- Sello de la Biblioteca del Instituto de 2. ${ }^{a}$ Enseñanza. Ávila].- Azpeitia (Guipúzcoa). Santuario de Loyola. 48-12626 [Exlibris de "J. Subirana, Barcelona".- Olim: 1-44-4].- Badajoz. Biblioteca Pública del Estado Bartolomé J. Gallardo. 4011 [Sello de la Biblioteca Provincial de Badajoz.- Sello del Instituto Provincial de Badajoz].- Barcelona. Ateneo. GO 610; Biblioteca de Cataluña. Enq. en pell [Sello: "J. Subirana ... Barcelona".Donación: Lluís Tusquets de Cabirol]; Enq. en mitja pell [Sello: "Biblioteca Particular de Miscelanea".- Donación: Lluís Tusquets de Cabirol]; Enq. en mitja pell [Donación: Lluís Tusquets de Cabirol]; Enq. en pell [Donación: Lluís Tusquets de Cabirol]; Biblioteca Pública Espiscopal del Seminario. 248.1 Fer [Sello: "Bibliotheca Episcopalis Seminarii Barcinonensis"], 268"18" Fer [Etiqueta: J. Subirana Puerta Ferrisa, 16 Barcelona]; Instituto de Bachillerato Jaime Balmes (Fondo depositado en la Universidad Pompeu Fabra. 860-191"18" [Sello: "Instituto Provincial de Segunda Enseñanza de Barcelona"].- Burgos. Archivo "Silveriano" de la Provincia Carmelitana O.C.D. Burgense. LIT 432 [Olim: Liter. A 48], [Sello de la Librería del Carmen de Burgos.- Sello de El Monte Carmelo, Revista Religiosa, 1 OCT. 1901, Madrid.- An. ms. "A mis amados padres, su hijo Antonio Vicent"]; Facultad de Teología del Norte de España. Xn 87 [Sello de la biblioteca del seminario Conciliar de S. Gerónimo de Burgos].Cádiz. Biblioteca Pública. XIX-1530 [Ex libris: "Libreria Nacional y Estrangera de Eduardo Gautier Calle de Sn. Francisco n ${ }^{\circ} 23$ Cadiz".- Olim: 4455].Cantoblanco (Madrid). Universidad Pontificia de Comillas. 4267141 [Sello seco de la Sociedad Católica Propagadora de Buenos Libros, Santander].- Granada. Biblioteca de Andalucía. ANT-XIX-183; Biblioteca Provincial de los Misiones Claretianos de Bética. P 443-C-2.- Guadalupe (Cáceres). Real Monasterio de Santa María de Guadalupe. L. 2163.- Huelva. Archivo Municipal. 19/R.7788 [Procede Fondo Díaz Hierro], 19/R.3427 [Procede Fondo Díaz Hierro].- León. Universidad de León, Facultad de Educación. ED693.- Madrid. Biblioteca Histórica Municipal. C/9008; Congregación de la Misión de San Vicente de Paúl. 113-C-36; Fundación Universitaria Española. PEN1/1230 [Ex-libris de Pedro Sainz Rodriguez y ex-libris ms. de José Abella]; Instituto Nacional de Gestión Sanitaria. 028813; Museo Nacional del Romanticismo. C-VI-30; Real Academia de Ciencias Morales y Políticas. 21015 [Sello de la Biblioteca del Legado Cárdenas.- An. ms.: Ded. del aut. en antep.]; Real Academia Española. 2-VII-76 [Sello de la Biblioteca de la Real Academia Española.- Sello de la Real Academia Española].- Segovia. Seminario Diocesano. 9956.- Sevilla. Archivo Municipal. 33- 
0163 [Sello de la Biblioteca Municipal de Sevilla]; Real Academia Sevillana de Buenas Letras. FA 2571 [Olim: 860-191 "18" FER].- Tarragona. Seminario Pontificio. 1.186.-Valencia. Real Colegio de las Escuelas Pías. Biblioteca de los Padres Escolapios. XIX/2820.- Zamora. Biblioteca Diocesana. 2835 [Sello en antep. de la "Biblioteca del Ecmo. Sr. Obispo Don L. F: Ortiz".- Ans. mss.: "Memoria a mi querido amigo el ... Dn. Luis Felipe Ortiz del encuadernador Manuel Ma de Zafra" , "R. 5880".- Sello de la "Biblioteca. Obispado de Zamora"].

4.5 Manual de Historia Natural, de Manuel María José de Galdo; para Secundaria y aprobado por R. O. 22 septiembre 1849, 26 septiembre 1850, 5 septiembre 1851, 15 septiembre 1852, 18 septiembre 1853, 13 octubre 1854, 1 octubre 1855, 13 septiembre 1858, 26 septiembre 1861, 31 agosto 1864, 14 septiembre 1867 y 1 agosto 1868.

Figura 16-17. Portada y detalle Manual de Historia Natural
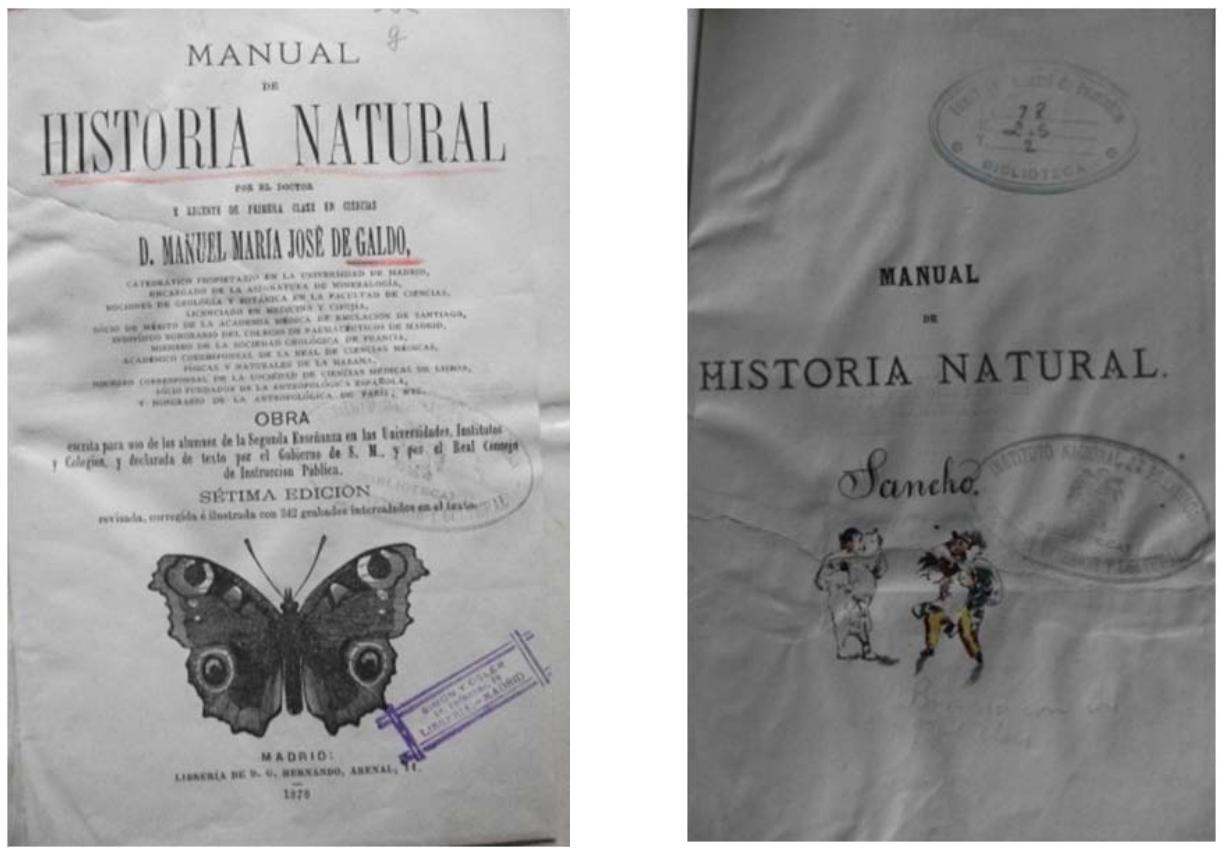
Figura 18. Interior de Manual de Historia Natural

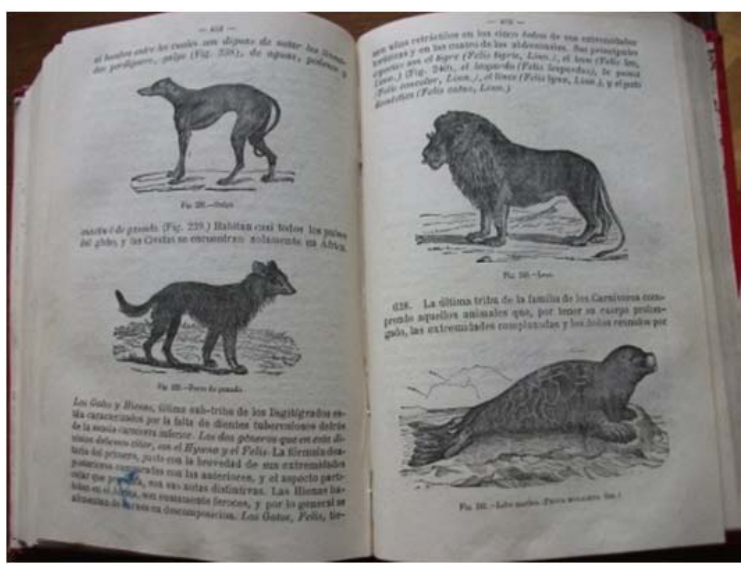

Galdo, Manuel María José de.

Manual de Historia Natural / por el doctor ... Manuel María José de Galdo.- $7^{\text {a }}$ ed. rev., corr. e il. con 342 grabados intercalados en el texto.- Madrid : Librería de D. G. Hernando, 1878 (Imprenta de Gregorio Hernando) VII, 622 p. : il ; 20 cm.

Procede de la Biblioteca Legado Sancho y en la anteportada figura F. Sancho Ferrando. La signatura que puede encontrarse en CCPB, 179702-6, es la antigua 021634, siendo la signatura actual 001264. Resalta por su grabados calcográficos y llama la atención las numerosas "ilustraciones" a color con motivos de animales (puede que sean calcomanías aunque es difícil de apreciar) con las que el propietario completó algunas de las páginas. Tiene ex-libris del INP.

Figura 19. Portada de Phitología

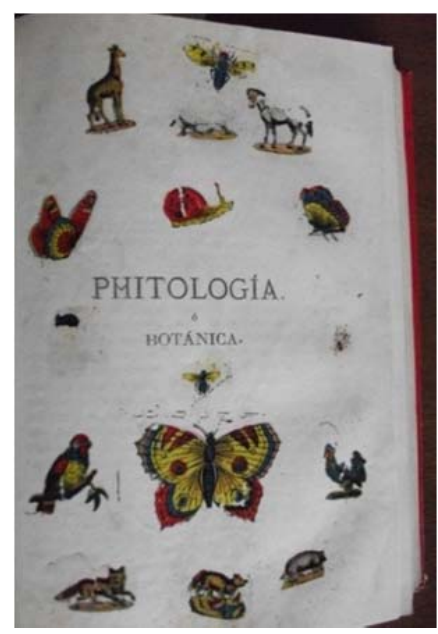

Figura 20. Portada de Zoología

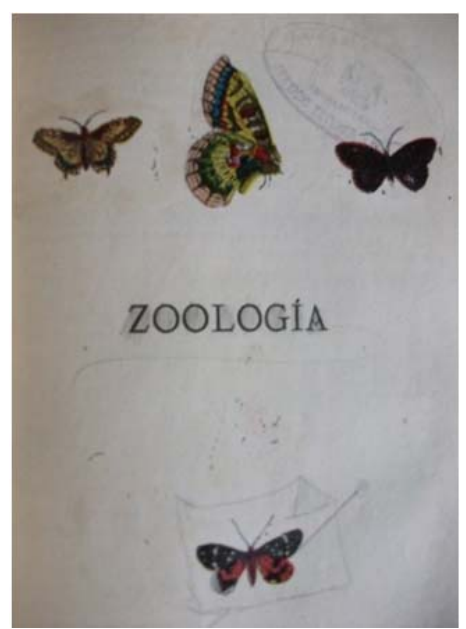


Las localizaciones según CCPB son: Albacete. Biblioteca Pública. A-502GAL-man [Sello de Instituto de Segunda Enseñanza de Albacete].- Cuenca. Seminario Mayor o Conciliar de San Julián. 148-E-18 [Falto de las últimas 159 p.].- Guadalupe (Cáceres). Real Monasterio de Santa María de Guadalupe. C. 397.- León. Biblioteca Regional Mariano Domínguez Berrueta. BRMB 5869 (*) [Sello de la Biblioteca Regional. Diputación. León.- Sello de la Librería y Encuadernación Antonio M. Cuesta. Salamanca].- Madrid. Instituto Nacional de Gestión Sanitaria. 021634 [Ex-libris del I.N.P.].- Pamplona. Instituto de Enseñanza Secundaria Plaza de la Cruz. A 3-1/12 [Olim: XR 25 B 66/3006].Santiago de Compostela (La Coruña). Instituto Teológico Compostelano. 1509.Zaragoza. Colegio de los Padres Escolapios, Biblioteca. 52-g-14.

4.6 Tratado de Aritmética de Mr. Lacroix, traducido por Rebollo, para Secundaria y aprobado por: R.O. 22 agosto 1846, 8 septiembre 1847, 14 septiembre 1848 y 22 septiembre 1849.

Figura 21. Portada de Aritmética

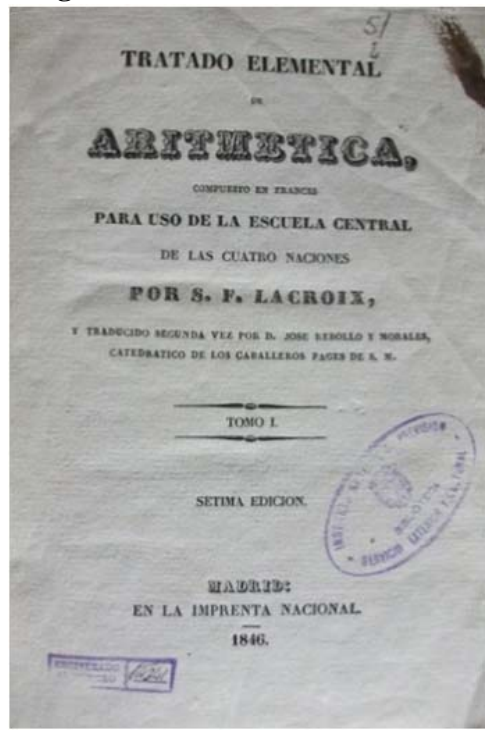

Figura 22. Dedicatoria

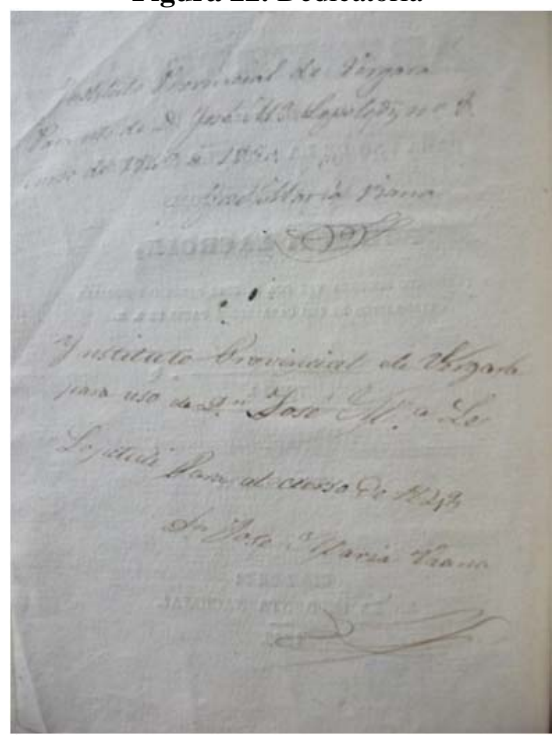

Lacroix, Silvestre-François (1765-1843)

Tratado elemental de aritmética : compuesto en francés para uso de la escuela Central de las Cuatro naciones / por S. F. Lacroix, y traducido segunda vez por Josef Rebollo y Morales.- $7^{\text {a }}$ ed. Madrid : Imprenta Nacional. 1846 XI, 368 p. ; 21 cm.- (Curso completo elemental de matemáticas puras ; I) 
Procede de la biblioteca del Duque de Bailén y en verso de la portada puede leerse, repetida, la anotación manuscrita "Instituto Provincial de Vergara. Para uso de D. José María Lopetedi, nº 8. Curso de 1849 a 1850. José María Viena”.

La signatura que puede encontrarse en CCPB, 338759-3, es la antigua 021640, siendo la signatura actual 001198.

Las localizaciones según CCPB son: Azpeitia (Guipúzcoa). Santuario de Loyola. 3071, 3-11.01.- Barcelona. Biblioteca Pública Episcopal del Seminario de Barcelona. 102545 [Anotaciones ms. a port.; sello: Vicente Oliva, Impresor y librero-Gerona.- Contiene únicamente t. I - R. 102545].- Burgos. Facultad de Teología del Norte de España. IV:87-23 [Etiqueta de la Biblioteca del Seminario de S. José de Burgos.- Contiene únicamente T. I.].- Cáceres. Biblioteca Pública del Estado "A. Rodríguez Moñino y María Brey". 2/7281 [Sello de la Biblioteca Provincial de Cáceres.- Sello de Vicente Paredes y Guillén, Arquitecto de Plasencia.-R. 3212] y 2/12954 [Sello de la Biblioteca Provincial de Cáceres.- Sello del Instituto de Segunda Enseñanza de Cáceres.- Contiene únicamente el vol. I].Cádiz. Biblioteca Provincial en Cádiz. XIX-1177.- Granada. Instituto de Bachillerato Padre Suárez. 1-48.- Ciudadela (Menorca). Seminario Diocesano de Menorca. 8446 [Contiene únicamente t. I].- Gerona. Seminario Diocesano de Gerona, Archivo y Biblioteca. 5/577 [Etiqueta de ex-libris: José Coll y Vehi].Huelva. Biblioteca Pública. F-1617.- Logroño. Instituto de Educación Secundaria Práxedes Mateo Sagasta. 511/LAC/tra [Ex-libris ms. de José Turrientes.Contiene únicamente t. I.- R. 8813].- Lugo. Biblioteca Pública. 3587/9.- Madrid. Biblioteca Central Militar (Instituto de Historia y Cultura Militar). VI-32-103(bis) [Sello y ex-libris de la Biblioteca de Ingenieros] y III-54-8-9[Ex-libris de la Biblioteca de Ingenieros del Ejército.- Sólo tomo I]; Fundación Universitaria Española. XIV/1060; Instituto Nacional de Gestión Sanitaria. 021640 [An. ms. en v. de port.: "Instituto Provincial de Vergara. Para uso de D. José $\mathrm{M}^{\mathrm{a}}$ Lopetedi... Jose Maria Viana"].- Mahón (Menorca). Ateneo Científico, Literario y Artístico de Mahón. A-1681 [Donado por Antonio Prieto.- Sólo t. 1].- Santiago de Compostela. Instituto Teológico Compostelano, Biblioteca. Sen sign. [Contiene solo t. I] y 9641.- Sevilla. Archivo Municipal de Sevilla.97-0060.

\section{CONCLUSIONES}

La Biblioteca del INGESA tiene un amplio y valioso Fondo Antiguo del que forman parte las Bibliotecas del Duque de Bailén, del Marqués de Riscal y el Legado de Sancho. Y en la misma se han podido localizar libros de texto autorizados por la legislación del momento para el estudio de alumnos de primaria y secundaria.

Rica la Biblioteca del INGESA, además de por el Fondo Antiguo por todo su fondo especializado en materia de Sanidad, llama la atención la no visibilidad 
directa en la web del Instituto, teniendo que acceder por el epígrafe Estadísticas y Estudios, que nada hace intuir que dentro esté el enlace de la biblioteca.

El cambio de signaturas no es un obstáculo para la consecución de los documentos una vez que se hace la petición en la propia biblioteca, pues los profesionales tienen los documentos perfectamente identificados, pero cuando la investigación se realiza "on line" la información que se localiza es diferente, lo que hace que esa diferencia vaya pasando de informe a informe como dato erróneo. Por ello, es preciso que cuando se produzca un ajuste de cualquier característica en materia de mejora operativa, la comunicación entre las bibliotecas y el ССРB debe ser inmediata para que todos los catálogos tengan la misma información.

\section{BIBLIOGRAFÍA}

BIBLIOTECA DEL INSTITUTO NACIONAL DE GESTIÓN SANITARIA (INGESA): Disponible en: www.ingesa.msssi.gob.es/Biblioteca/home.htm [Consulta: 30/10/2014]. http://bvingesa.msc.es/bvingesa/es/consulta/busqueda. cmd [Consulta: 30/10/2014]. www.ingesa.msssi.gob.es/Biblioteca/docs/ InformacionGeneral.pdf [Consulta: 30/10/2014].

CAMPO PUERTA, $\mathrm{M}^{\mathrm{a}}$ Pilar del (2015). Materiales didácticos de los siglos XVIII y XIX en bibliotecas madrileñas. Tesis doctoral. Madrid: UCM.

CHOPPIN, Alain (1992) Les manuels scolaires: histoire et actualité, París: Hachette.

ESPAÑA. Ministerio de Fomento. Boletín oficial del Ministerio de Fomento, de 6 noviembre de 1856, n 254, Año V. Tomo XX, p. 239.

ESPAÑA. Ley de Instrucción Pública, de 9 de septiembre de 1857. En Colección Legislativa de España, tomo LXXIII, pp. 256-305, en "Historia de la educación en España", tomo II, De las Cortes de Cádiz a la revolución de 1868. Ministerio de Educación, Libros de Bolsillo de la Revista de Educación. Madrid, 1979. Disponible en: http://personal.us.es/alporu/historia/ley_moyano_texto.htm [Consulta: 30/10/2014].

ESPAÑA. Ley 16/1985, de 25 de junio de Patrimonio Histórico Español. Boletín Oficial del Estado, de 29 de junio de 1985, no 155, pp. 20342-20352. Disponible en: www.boe.es/diario_boe/txt.php?id=BOE-A-1985-12534 [Consulta: 30/10/2014].

INSTITUTO NACIONAL DE GESTIÓN SANITARIA (INGESA): Disponible en: www.ingesa.msc.es/organizacion/quienes/home.htm [Consulta: 30/10/2014].

LOPEZ DEL CASTILLO, $\mathrm{M}^{\mathrm{a}}$ Teresa (1982) "Planes y programas escolares en la legislación española” en BORDÓN $n^{\circ}$. 242-243. Madrid, Sociedad Española de Pedagogía.

MINISTERIO de Educación (1979) "Colección Legislativa de Instrucción Primaria. Madrid, 1856” en Ministerio de Educación y Ciencia (1979) Historia 
de la Educación en España. Textos y Documentos. Vol II: De las Cortés de Cádiz a la revolución de 1868. Madrid: Ministerio de Educación.

MINISTERIO de Educación y Ciencia (1985) HISTORIA de la Educación en España. Vol I: Del despotismo ilustrado a las Cortes de Cádiz. Madrid: Ministerio de Educación y Ciencia.

MINISTERIO de Educación y Ciencia (1985) Historia de la Educación en España. Textos y Documentos. Vol II: De las Cortés de Cádiz a la revolución de 1868. Madrid: Ministerio de Educación.

PLAN GENERAL DE INSTRUCCIÓN PÚBLICA. Disponible en: www.filosofia.org/mfa/fae836a.htm\#00 [Consulta: 30/10/2014].

PLAN GENERAL DE INSTRUCCIÓN PÚBLICA. Real Decreto de 17 de septiembre de 1845. Disponible en:www.filosofia.org/mfa/fae845a.htm [Consulta: 30/10/2014].

TIANA FERRER, Alejandro (1999) "La investigación histórica sobre los manuales escolares en España: el proyecto MANES” en Clio \& Asociados. La historia enseñada, $\mathrm{n}^{\circ}$ 4, pp. 101-119.

VILLALAÍ́N BENITO, José Luis (1999) Manuales escolares en España: Tomo I: Legislación (1812-1939). Madrid: Universidad Nacional de Educación a Distancia.

VILLALAÍN BENITO, José Luis (1999) Manuales escolares en España: Tomo II: Libros de textos autorizados y censurados (1833-1874). Madrid: Universidad Nacional de Educación a Distancia.

VILLALAÍN BENITO, José Luis (1999) Manuales escolares en España: Tomo III: Libros de textos autorizados y censurados (1874-1939). Madrid: Universidad Nacional de Educación a Distancia. 